\title{
Sampling plans using extended EWMA statistic with and without auxiliary information
}

\author{
Muhammad Naveed ${ }^{1}$, Muhammad Azam², Muhammad Saeed ${ }^{3}$, Nasrullah Khan ${ }^{4}$, \\ Muhammad Aslam ${ }^{*}$ \\ ${ }^{1}$ Dept. of Statistics, National College of Business Administration and Economics, \\ Lahore, 54660, Pakistan. \\ ${ }^{2}$ Dept. of Statistics and Computer Science, University of Veterinary and Animal \\ Sciences, Lahore, 54000, Pakistan. \\ ${ }^{3}$ Dept. of Statistics, Minhaj University, Lahore,54770, Pakistan. \\ ${ }^{4}$ Dept. of Statistics, College of Veterinary and Animal Sciences (Jhang Campus), \\ Jhang 35200, Pakistan. \\ ${ }^{5}$ Dept. of Statistics, Faculty of Science, King Abdulaziz University, Jeddah 21551, \\ Saudi Arabia. \\ Correspondingauthor:aslam_ravian@hotmail.com
}

\begin{abstract}
In this paper, we propose an Acceptance Sampling Plan (ASP) using the statistic suggested by Naveed et al. (2018) under the condition of known and unknown population standard deviation (SD) for the presence of with and without Auxiliary Information (AI). It is presumed that the study variable of quality trait and AI follow the bivariate normal distribution. The plan parameters of the recommended plan are discussed for all four cases under the constraint that specified producer and consumer risks are gratified. The suggested plan is compared in terms of sample size (SS) with numerous existing plans and showed that the presented plan has a smaller SS for any value of AQL, LQL. Various tables of plan parameters have been erected using various combinations of smoothing constants for industrial use. For the workable purpose, the industrial example has also been examined. At last, concluding remarks are discussed.
\end{abstract}

Keywords: Acceptance number; acceptance sampling plan; auxiliary information; extended EWMA; sample size.

\section{Introduction}

As buyer trust the specification mentioned very obligatory for the manufacturer to by the manufacturer on their products. It's produce the product according to the 
mentioned specification limits. Good experience of buyers enhances the reputation of the product. In practice, it is very difficult to inspect the peculiarity of every product because a lot of time is consumed in this exercise, and cost is also increased. So, to overwhelm this hurdle a device used in statistical quality control (SQC) is known as an acceptance sampling plan (ASP). ASPs ensure both the purchaser and seller a decision rule to acknowledge the lot or discard it based on sample data taken from the lot. ASPs are comprehensively practiced in assembling industries to scrutinize the crude material, in-process and finished items. There are so many types of plans which are practiced in inspection of the lot, like single, double, repetitive, multiple, etc. among them single sampling plan (SSP) is quite familiar owing to its ease of implementation. A lot of work can be seen in the literature using SSP like Lieberman \& Resnikoff (1955) constructed various tables for parametric values of SSP using various acceptance quality level (AQL) under the condition of MIL-STD 414 scheme. After that Owen (1967) proposed SSP for normal distribution (ND) under the condition of unknown process standard deviation (SD). Hamaker (1979) suggested the method for finding the values of the parameter in SSP under the scenario of unknown SD. Two foremost types of ASPs are attribute and variable sampling plans. Collani (1991) had shown that attribute plans are more appropriate if the buyer has concerned with fraction non-conforming in lots. But later on Seidel (1997) has shown that a variable sampling plan (VSP) is always optimum. More work using VSP can be viewed in S Balamurali \& Jun (2006), Saminathan Balamurali \& Jun (2007), Klufa (2010), Aslam et al. (2011), Wang \& Tsai (2012), Al-Omari \& Al-Hadhrami (2018), Cha \& Badía (2020), Chakrabarty et al. (2020), Tripathi et al. (2020), Yemshanov et al. (2020)

The utilization of memory type statistic in the field of ASPs has attained much more consideration in recent past owing to his eminent performance in determining the lesser values of its plan parameters. Aslam et al. (2015b) suggested an SSP based on exponentially weighted moving average (EWMA) statistic and calculate the parametric values of SSP. The results are likening to the memoryless plan which shows the far better evaluation in the form of lesser values of its parameters. The authors also proved that the recommended plan achieved much better results in comparison to the plans presented by 
Saminathan Balamurali \& Jun (2007) and Aslam et al. (2013). Yen et al. (2014) presented an SSP based on EWMA statistic using yield index and exhibit the lesser values of sample size (SS) as collating it to the memoryless yield index plan. After that Aslam et al. (2015a) improved the result of EWMA based SSP using repetitive sampling (RS). Khan et al. (2018) presented another memory-based ASP using modified EWMA statistic and showed a far better results when compared with two existing plans.

The use of auxiliary information (AI) in ASP is very beneficial in terms of reducing the time as well as the cost of the inspection. The concept of AI in ASP has been introduced by Aslam et al. (2017) and showed the lesser value of SS using AI. later on Aslam et al. (2018) used a memory type regression estimator in ASP to enhance the capability of the recommended plan in terms of smaller values of parameters. More work on ASPs using AI can be seen in Azam et al. (2016), Khan et al. (2019), motivated by the use of memory-based information as well as AI in ASPs, we present an ASP using memory-based statistic presented by Naveed et al. (2018) with and without AI. The remaining paper is described as: in Section 2 , the designing of the recommended plan without AI has been discussed. In section 3, the construction of the suggested plan using AI has been examined. In section 4, comparability of the presented plan has been discussed with the existing plan. In Section 5, the industrial application has been examined. In the last Section concluding remarks are given.

\section{Designing of the proposed plan without}

\section{AI}

Naveed et al. (2018) presented a memorybased statistic for the quality trait of Normal Distribution (ND) named as Extended EWMA statistic which is given as

$\mathrm{W}_{\mathrm{i}}=\tau_{1} \overline{\mathrm{Z}}_{\mathrm{i}}-\tau_{2} \overline{\mathrm{Z}}_{\mathrm{i}-1}+\left(1-\tau_{1}+\tau_{2}\right) \mathrm{W}_{\mathrm{i}-1}$

Such that $0<\tau_{1} \leq 1$ and $0 \leq \tau_{2}<\tau_{1}$. Where $\tau_{1}$ and $\tau_{2}$ are smoothing constants. Like traditional EWMA statistic the performance of initiated statistic is quite well for smaller values of smoothing constants. The proposed statistic is the generalization of Roberts (1959) and converted to EWMA statistic when $\tau_{2}=0$. The intended statistic is an unbiased estimator of process mean $\mu$ with variance

$$
\begin{aligned}
& \operatorname{var}\left(\mathrm{W}_{\mathrm{i}}\right)=\frac{\sigma^{2}}{m}\left[\left(\tau_{1}{ }^{2}+\tau_{2}{ }^{2}\right)\left\{\frac{1-r^{2 i}}{1-r^{2}}\right\}-\right. \\
& \left.2 \mathrm{r} \tau_{1} \tau_{2}\left\{\frac{1-r^{2 i-2}}{1-r^{2}}\right\}\right]
\end{aligned}
$$

Where $r=1-\tau_{1}+\tau_{2}$. 
The above expression of variance is reduced to Roberts (1959) the expression if we put $\tau_{2}=0$. It is to be remembered that $\tau_{2}<\tau_{1}$. The variance expression of the proposed statistic will give a minimum result if we take the value of $\tau_{2}$ is nearer to $\tau_{1}$. For example if $\tau_{1}=0.1$ then for any value within the range of $\tau_{2}\left(0 \leq \tau_{2}<\tau_{1}\right)$ will give the smaller variance result than EWMA variance, but if we take $\tau_{2}=0.09$ than the result of the proposed variance is smallest for any other values of $\tau_{2}$.

Now we will construct a sampling plan based on recommended idea under two different scenarios

- When process variability is known

- When process variability is not known

\subsection{When process variability is known}

We present the succeeding recommended plan for variable suggested by Naveed et al. (2018):

Step 1: Firstly, we chose a sample of size $m$ from the running lot at the time $\mathrm{i}$ and calculate $\bar{Z}_{i}$, the average value of the study variable. After that, we utilized it to find out the value of offered statistic using predefine values of $\tau_{1}$ and $\tau_{2}$ as

$\mathrm{W}_{\mathrm{i}}=\tau_{1} \overline{\mathrm{Z}}_{\mathrm{i}}-\tau_{2} \overline{\mathrm{Z}}_{\mathrm{i}-1}+\left(1-\tau_{1}+\tau_{2}\right) \mathrm{W}_{\mathrm{i}-1}$

Step 2: After getting the value of $W_{i}$, calculate the statistic $\mathrm{M}_{\mathrm{i}}$ as follows
$\mathrm{M}_{\mathrm{i}}=\frac{\mathrm{USL}-\mathrm{W}_{\mathrm{i}}}{\sigma}$

If the value of $M_{i} \geq L_{a}$, we accept the current lot, contrarily we discard the running lot. In the first step, while we calculate the value of the presented statistic, we set the value of $\overline{\mathrm{Z}}_{0}$ and $\mathrm{W}_{0}$ is zero at $i=1$. The prospective plan is converted to Aslam et al. (2015b) when $\tau_{2}=0$, and reduced to a conventional variable single plan if $\tau_{1}=$ 1 and $\tau_{2}=0$. it is experienced that when the engineers apply this type of plan repeatedly, the effect of time index $i$ is very low which is negligible. That's why we omit the time index factor invariance expression of the recommended statistic. The two parameters of the suggested plan named as the sample size (SS) denoted by $m$ and acceptance number (AN) designated by $L_{a}$.

The operating characteristic (OC) function of the presented plan when $p$ is the proportion of nonconformist is extracted as follows

$$
\begin{aligned}
& P_{a}(p)=P\left(\mathrm{M}_{\mathrm{i}} \geq L_{a} \mid p\right)=P\left(\mathrm{~W}_{\mathrm{i}}+L_{a} \sigma \leq\right. \\
& U S L \mid p)
\end{aligned}
$$

According to Duncan (1986), we have

$\mathrm{W}_{\mathrm{i}}+L_{a} \sigma \sim N\left(\mu+L_{a} \sigma, \frac{\sigma^{2}}{m}\left[\frac{\tau_{1}{ }^{2}+\tau_{2}{ }^{2}-2 \mathrm{r} \tau_{1} \tau_{2}}{1-r^{2}}\right]\right)$ where $r=1-\tau_{1}+\tau_{2}$

After simplification of the above equation (5), the final form of OC function under the 
condition of known standard deviation (SD) is given as

$P_{a}(p)=\Phi\left(\left(Z_{p}-L_{a}\right) \sqrt{\frac{m}{\left[\frac{\tau_{1}^{2}+\tau_{2}^{2}-2 \tau_{1} \tau_{2}}{1-r^{2}}\right]}}\right)$

Here $\Phi($.$) is the cumulative distribution$ function (CDF) and $Z_{p}$ is the $p t h$ percentile of standard normal distribution (SND).

Two risks are involved while we making a decision about the lot. One is the producer's risk designated by $\alpha$ is the probability of discarding the correct the lot and other is consumer risk expressed by $\beta$ is the probability of accepting the defective lot. Every manufacture wishes that the probability of lot accepting (PLA) should be larger than $(1-\alpha)$ at an acceptable quality level (AQL). Perversely, every client wants that PLA should be smaller than $\beta$ at the limiting quality level (LQL). The parametric values of the initiated plan will be determined under the condition that both risks are gratified concurrently. During the simulation experience, we noticed that there

1. If the values of AQL and LQL are fixed, the SS $m$ increases as the value of the smoothing constant is increased. For example, when $A Q L=0.001$ and $L Q L=0.002$, then the value of $m=3 \operatorname{using} \tau_{1}=$ $0.1, \tau_{2}=0.09$. For $\tau_{1}=0.3, \tau_{2}=$ are so many combinations of SS and AN satisfy the conditions, from these combinations we selected that pair of combination which has the smallest value of SS. The parametric values of the offered plan are determined such that the OC curve must pass the points $(\alpha, A Q L)$ and $(\beta, L Q L)$. we will utilize the subsequent non-linear optimization to find the parametric values of the initiated plan:

minimize $m$

With the condition that

$P_{a}(A Q L)=\Phi\left(\left(Z_{p}-L_{a}\right) \sqrt{\frac{m}{\sqrt{\frac{\tau^{2}+\tau_{2}-2-2 r_{1} \tau_{2}}{1-r^{2}}}}}\right) \geq 1-\alpha$

And

$P_{a}(L Q L)=\Phi\left(\left(Z_{p}-L_{a}\right) \sqrt{\frac{m}{\left[\frac{\tau_{1}{ }^{2}+\tau_{2}{ }^{2}-2 \tau_{1} \tau_{2}}{1-r^{2}}\right]}}\right) \leq \beta$

The parametric values of the proposed plan when SD is known are placed in Table 1 for numerous levels of AQL, LQL, and distinct values of smoothing constants.

0.29, $\mathrm{m}=18$, For $\tau_{1}=0.5, \tau_{2}=$ 0.49 , the value of SS is 51 .

2. For fixed values of smoothing constants and AQL, we observe the diminishing trend in SS $m$ as LQL increases. For example, when $\tau_{1}=0.5, \tau_{2}=0.49$ and AQL $=$ 


$$
\begin{aligned}
& 0.005, \text { then for }=0.010, m= \\
& 37, \quad=0.015, m=14, \quad= \\
& 0.020, m=9, \quad=0.030, \quad= \\
& 5, \quad=0.040, m=4
\end{aligned}
$$

\subsection{When process variability is not known}

In this section, we discuss the scenario when its process SD is not known and we estimate it with the help of sample data. The first step of the recommended plan is the same as for the known case in section 2.1, but in the second step we use sample standard deviation (SD) $S$ instead of $\sigma$ in statistic $M_{i}$ i.e

$\mathrm{M}=$

Table 1. The plan parameters of suggested plan when SD is known

\begin{tabular}{|r|r|r|r|r|r|r|r|}
\hline & & $=0.1$ & $=0.09$ & $=0.3$ & $=0.29$ & $=0.5$ & $=0.49$ \\
\cline { 3 - 8 } AQL & LQL & & & & & & \\
\hline 0.001 & 0.002 & 3 & 2.9665 & 18 & 2.9704 & 51 & 2.9701 \\
\hline & 0.004 & 2 & 2.8339 & 5 & 2.8367 & 12 & 2.8476 \\
\hline & 0.006 & 2 & 2.8307 & 3 & 2.7514 & 7 & 2.7639 \\
\hline & 0.008 & 2 & 2.6385 & 2 & 2.7127 & 5 & 2.7124 \\
\hline 0.005 & 0.010 & 3 & 2.4543 & 14 & 2.4327 & 37 & 2.4325 \\
\hline & 0.015 & 2 & 2.3532 & 5 & 2.3513 & 14 & 2.3484 \\
\hline & 0.020 & 2 & 2.1688 & 4 & 2.2592 & 9 & 2.2770 \\
\hline & 0.030 & 2 & 2.3776 & 2 & 2.1589 & 5 & 2.2048 \\
\hline 0.03 & 0.06 & 2 & 1.7119 & 8 & 1.6934 & 22 & 1.6977 \\
\hline & 0.09 & 2 & 1.4527 & 3 & 1.5763 & 8 & 1.5768 \\
\hline & 0.12 & 2 & 1.3231 & 2 & 1.4630 & 5 & 1.4941 \\
\hline & 0.15 & 2 & 1.2841 & 2 & 1.5079 & 4 & 1.4266 \\
\hline & 0.10 & 2 & 1.4271 & 7 & 1.4381 & 17 & 1.4396 \\
\hline 0.05 & 0.15 & 2 & 1.3050 & 3 & 1.2784 & 6 & 1.3028 \\
\hline & 0.20 & 2 & 1.1856 & 2 & 1.1593 & 4 & 1.1792 \\
\hline & 0.25 & 2 & 0.8364 & 2 & 1.0855 & 3 & 1.1417 \\
\hline & & & & & & &
\end{tabular}

Where $S=\sqrt{\frac{\sum_{i=1}^{m}\left(Z_{i}-\bar{Z}_{\mathrm{i}}\right)}{}}$

We reject the lot if $\mathrm{M}<\mathrm{L}$ and accept it if M L

The OC function for the unknown case is derived as

$$
=P(\mathrm{M} \quad)=P\left(\mathrm{~W}_{\mathrm{i}}+\quad\right)
$$

According to (Duncan, 1986), we have

$$
\mathrm{W}_{\mathrm{i}}+\underset{\quad \operatorname{ar}(S))}{\left(\mu+E(S), \operatorname{Var}\left(\mathrm{W}_{\mathrm{i}}\right)+\right.}
$$

As we

know that $E(S)=\quad \operatorname{ar}(S)=(1 \quad) \sigma$ 
where $c_{4}=$

$\sqrt{[2 /(m-1)]} \Gamma(m / 2) / \Gamma[m-1 / 2]$

these results are used by various researches

like Aslam et al. (2015b), Aslam et al. (2015a), Azam et al. (2016), Aslam et al. (2018),

So

$\mathrm{W}_{\mathrm{i}}+L_{a} S \sim N\left(\mu+L_{a} \sigma c_{4},\left[\frac{\tau_{1}{ }^{2}+\tau_{2}{ }^{2}-2 \mathrm{r} \tau_{1} \tau_{2}}{1-r^{2}}\right] \frac{\sigma^{2}}{m}+\right.$

$\left.L_{a}^{2}\left(1-c_{4}^{2}\right) \sigma^{2}\right)$

where $r=1-\tau_{1}+\tau_{2}$

The final form of OC function is given as

$P_{a}(p)=\Phi\left(\left(Z_{p}-L_{a} c_{4}\right) \sqrt{\frac{1}{\left[\frac{\tau_{1}{ }^{2}+\tau_{2}{ }^{2}-2 \mathrm{r} \tau_{1} \tau_{2}}{1-r^{2}}\right] \frac{1}{m}+L_{a}^{2}\left(1-c_{4}^{2}\right)}}\right)$

The optimization problem for the case of unknown $\mathrm{SD}$ is given as

minimize $m$

With respect to

$P_{a}(A Q L)=$

$\Phi\left(\left(Z_{p}-L_{a} c_{4}\right) \sqrt{\frac{1}{\left[\frac{\tau_{1}{ }^{2}+\tau_{2}{ }^{2}-2 \mathrm{r} \tau_{1} \tau_{2}}{1-r^{2}}\right] \frac{1}{m}+L_{a}^{2}\left(1-c_{4}^{2}\right)}}\right) \geq 1-\alpha$

And

$$
\begin{aligned}
& P_{a}(L Q L)= \\
& \Phi)\left(Z_{p}-\right.
\end{aligned}
$$

$$
\left.\left.L_{a} c_{4}\right) \sqrt{\frac{1}{\left[\frac{\tau_{1}^{2}+\tau_{2}{ }^{2}-2 \mathrm{r} \tau_{1} \tau_{2}}{1-r^{2}}\right] \frac{1}{m}+L_{a}^{2}\left(1-c_{4}^{2}\right)}}\right) \leq \beta
$$

We used grid search method to calculate the SS and AN. The parametric values $m$ and $L_{a}$ of the proposed plan for unknown SD are reported in Table 2 using numerous values of AQL, LQL, and smoothing constants. From Table 2, we perceive the same pattern in the values of parameters as we noticed in the known case. However, we see that SS is much large for the unknown case. For example, when $A Q L=$ 0.03 and $L Q L=0.06$ the value of $m$ is 119 for an unknown case and it was just 2 for a known case when $\tau_{1}=0.1$ and $\tau_{2}=0.09$.

\section{Designing of the proposed plan using} AI

The utilization of AI has always beneficial in enhancing the precision of decisions. So, in this section, we consider that some extra information that is correlated to the study variable is given. In this section, we will discuss the suggested plan for the case of known and unknown SD. 
Table 2. The plan parameters of suggested plan when SD is unknown

\begin{tabular}{|c|c|c|c|c|c|c|c|}
\hline \multirow{2}{*}{} & & $=0.1$ & $=0.09$ & $=0.3$ & $=0.29$ & $=0.5$ & $=0.49$ \\
\cline { 3 - 7 } & & & & & & & \\
\hline 0.001 & 0.005 & 129 & 2.8064 & 131 & 2.8060 & 137 & 2.8057 \\
\hline & 0.007 & 82 & 2.7429 & 83 & 2.7423 & 87 & 2.7418 \\
\hline & 0.009 & 61 & 2.6922 & 61 & 2.6940 & 65 & 2.6947 \\
\hline & 0.012 & 44 & 2.6366 & 45 & 2.6346 & 47 & 2.6375 \\
\hline & 0.016 & 33 & 2.5787 & 34 & 2.5729 & 35 & 2.5779 \\
\hline 0.006 & 0.015 & 200 & 2.3227 & 207 & 2.3223 & 219 & 2.3224 \\
\hline & 0.017 & 150 & 2.2950 & 154 & 2.2950 & 164 & 2.2946 \\
\hline & 0.020 & 107 & 2.2591 & 109 & 2.2599 & 117 & 2.2592 \\
\hline & 0.025 & 71 & 2.2087 & 72 & 2.2094 & 77 & 2.2093 \\
\hline & 0.030 & 52 & 2.1666 & 55 & 2.1644 & 57 & 2.1676 \\
\hline 0.03 & 0.055 & 162 & 1.7246 & 171 & 1.7247 & 188 & 1.7243 \\
\hline & 0.057 & 143 & 1.7155 & 150 & 1.7153 & 165 & 1.7146 \\
\hline & 0.059 & 126 & 1.7054 & 133 & 1.7051 & 147 & 1.7056 \\
\hline & 0.06 & 119 & 1.7013 & 126 & 1.7006 & 138 & 1.7006 \\
\hline & 0.09 & 39 & 1.5887 & 41 & 1.5855 & 46 & 1.5883 \\
\hline 0.05 & 0.08 & 174 & 1.5123 & 186 & 1.5123 & 210 & 1.5122 \\
\hline & 0.09 & 104 & 1.4770 & 111 & 1.4777 & 126 & 1.4774 \\
\hline & 0.10 & 70 & 1.4465 & 76 & 1.4472 & 85 & 1.4450 \\
\hline & 0.11 & 51 & 1.4177 & 55 & 1.4154 & 62 & 1.4156 \\
\hline & 0.15 & 22 & 1.3166 & 23 & 1.3175 & 27 & 1.3173 \\
\hline
\end{tabular}

3.1 When population SD is known 8

The initiated plan using AI for the case of known SD is stated as follows:

Step-1: Select a bivariate sample of size mmon $\left(x_{i}, t_{i}\right)$ from running a lot and compute the

regression estimate as

$$
=\bar{x}+\left(\mu_{t}-\bar{t}\right)
$$

where $=-r, S$ are sample standard deviations of the main variable and $\mathrm{AI}$ respectively, and $r$ is the sample correlation between two variables. It is presumed that these variables are linearly related and regression line does not pass through the

origin. It is also supposed that the population mean of $t_{i}, \mu_{t}$ is known. The mean and variance of the regression estimator are 
$E\left(\operatorname{Reg}_{i}\right)=\mu_{x}=\mu$

$\operatorname{var}\left(\operatorname{Reg}_{i}\right)=\frac{\sigma_{x}}{m m}\left(1-\rho^{2}\right)$

After obtaining the value of $\operatorname{Reg}_{i}$ we calculate the value of EEWMA statistic using smoothing constant $\tau_{1}$ and $\tau_{2}$ as

EEWMA $=$ $+$

$\left(1-\tau_{1}+\tau_{2}\right)$ EEWMA $_{\mathrm{i}-1}$

Step-2: Calculate the following statistic as $=$

Accept the lot if $M \operatorname{Reg} g_{i} \geq J_{a}$ and reject the lot if $M \operatorname{Reg} g_{i}<J_{a}$. The presented plan has two parameters named as SS $\mathrm{mm}$ and $\mathrm{AN} J_{a}$. We use the value of and EEWMA is zero at $i=1$. The suggested plan is the generalization of numerous existing plans. When $\rho=0$, this plan gives the same results as discussed in Section 2.1. If $\tau_{2}=0$, the recommended plan is converted to Aslam et al. (2018). If $=0 \quad=0$, the suggested plan is transformed to Aslam et al., (2015b), if $=1=0$, the presented plan is converted to Aslam et al. (2017), when $=1, \quad=0$ and $=0$, the proposed plan is converted to traditional variable ASP. It is to be noted that for a large value of ,EEWMA approximately purse the normal distribution with parameters:EEWMA

$$
(\mu,-(1 \quad))
$$

The OC function of the proposed plan can be derived as

$$
\begin{gathered}
=P\left(\operatorname{MReg}_{i} \geq J_{a}\right)=P(\text { EEWMA }+ \\
U S L)
\end{gathered}
$$

According to Duncan (1986), we have

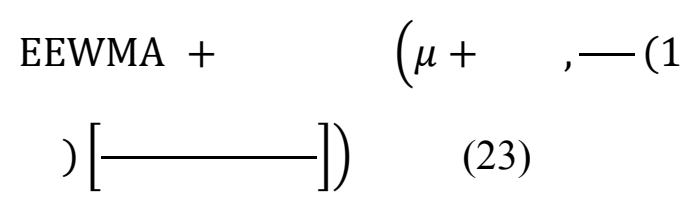

After simplification of the above equation, the final form of $\mathrm{OC}$ function under the condition of known standard deviation (SD) is given as

$$
(p)=
$$

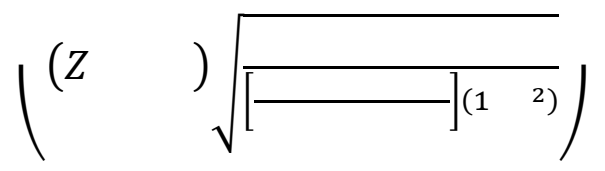

Here $\Phi($.$) is the cumulative distribution$ function (CDF) and $Z_{p}$ is the $p$ th percentile of standard normal distribution (SND).

Now we will utilize the following two optimizations to find the parametric values of the initiated plan:

minimize $\mathrm{mm}$

With the condition that

$$
(A Q L)=
$$

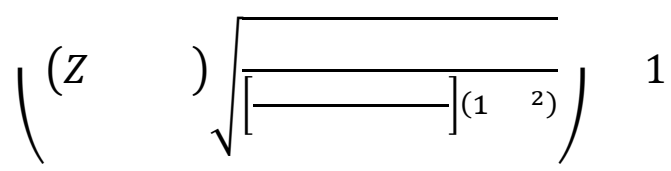


and

$$
\begin{aligned}
& P_{a}(L Q L)= \\
& \Phi\left(\left(Z_{p}-J_{a}\right) \sqrt{\frac{m m}{\left[\frac{\tau_{1}^{2}+\tau_{2}^{2}-2 \mathrm{r} \tau_{1} \tau_{2}}{1-r^{2}}\right]\left(1-\rho^{2}\right)}}\right) \leq \beta
\end{aligned}
$$

\subsection{When population SD is not known}

Here, we discuss the working operation of the suggested plan with the presence of AI when population SD is not known. The proceeding of step-1 remains the same as mentioned in section 3.1 for known SD. Later on in step-2, we use sample SD in acceptance statistic instead of $\sigma$ i.e

$$
\begin{aligned}
& M \operatorname{Meg}_{i}=\frac{U S L-\mathrm{EEWMA}_{\mathrm{i}}}{S_{x}} \\
& \text { where } S=\sqrt{\frac{\sum_{i=1}^{m}\left(x_{i}-\overline{\mathrm{x}}_{\mathrm{i}}\right)^{2}}{m-1}}
\end{aligned}
$$

The lot is rejected if $M \operatorname{Reg} g_{i}<\mathrm{J}_{\mathrm{a}}$ otherwise we accept it.

The OC function of the proposed plan for the unknown case using $\mathrm{AI}$ is derived as $P_{a}=P\left(\right.$ MReg $\left._{i} \geq J_{a}\right)=P\left(\right.$ EEWMA $_{\mathrm{i}}+$ $\left.J_{a} S_{x} \leq U S L\right)$

According to Duncan (1986), we have $\mathrm{EEWMA}_{\mathrm{i}}+J_{a} S_{x} \sim$ $N\left(\mu+J_{a} E\left(S_{x}\right), \operatorname{Var}\left(\mathrm{EEWMA}_{\mathrm{i}}\right)+\right.$ $\left.J_{a}^{2} \operatorname{Var}\left(S_{x}\right)\right)$ As we know that $E\left(S_{x}\right)=\sigma c_{4}$ and $\operatorname{Var}\left(S_{x}\right)=\left(\sigma^{2}-\sigma^{2} c_{4}^{2}\right)$ where $c_{4}=$

$\sqrt{[2 /(m-1)]} \Gamma(m / 2) / \Gamma[m-1 / 2]$

So

$\operatorname{EEWMA}_{\mathrm{i}}+L_{a} S \sim N\left(\mu+J_{a} \sigma c_{4},\left(1-\rho^{2}\right)\left[\frac{\tau_{1}{ }^{2}+\tau_{2}{ }^{2}-2 \mathrm{rr}_{1} \tau_{2}}{1-r^{2}}\right] \frac{\sigma^{2}}{m m}+\right.$

$\left.J_{a}^{2}\left(\sigma^{2}-\sigma^{2} c_{4}^{2}\right)\right)$

Where $r=1-\tau_{1}+\tau_{2}$

The final form of OC function is given as

$$
\begin{aligned}
& P_{a}(p)= \\
& \Phi\left(\left(Z_{p}-\right.\right. \\
& \left.\left.J_{a} c_{4}\right) \sqrt{\frac{1}{\left(1-\rho^{2}\right)\left[\frac{\tau_{1}{ }^{2}+\tau_{2}{ }^{2}-2 \mathrm{r}_{1} \tau_{2}}{1-r^{2}}\right] \frac{1}{m m}+J_{a}^{2}\left(1-c_{4}^{2}\right)}}\right)
\end{aligned}
$$

The optimization problem for the case of unknown SD is given as

$$
\text { minimize } \mathrm{mm}
$$

With respect to

$$
\begin{aligned}
& P_{a}(L Q L)= \\
& \Phi\left(\left(Z_{p}-\right.\right. \\
& \left.L_{a} c_{4}\right) \sqrt{\left.\frac{\left(1-\rho^{2}\right)\left[\frac{\tau_{1}{ }^{2}+\tau_{2}{ }^{2}-2 \tau_{1} \tau_{2}}{1-r^{2}}\right] \frac{1}{m m}+L_{a}^{2}\left(1-c_{4}^{2}\right)}{(33)}\right) \leq} \\
& \beta \quad \text { And }
\end{aligned}
$$




$$
\begin{aligned}
& P_{a}(A Q L)= \\
& \Phi\left(\left(Z_{p}-\right.\right. \\
& \left.\left.J_{a} c_{4}\right) \sqrt{\frac{1}{\left(1-\rho^{2}\right)\left[\frac{\tau_{1}^{2}+\tau_{2}{ }^{2}-2 \tau_{1} \tau_{2}}{1-r^{2}}\right] \frac{1}{m m}+J_{a}^{2}\left(1-c_{4}^{2}\right)}}\right) \geq \\
& 1-\alpha
\end{aligned}
$$

The values of parameters $m m$ and $J_{a}$ are placed in Tables 3-6 using different values of $\tau_{1}, \tau_{2}$ and $\rho$ for various levels of AQL and LQL under the condition of known and unknown SD. The behavior of $\mathrm{mm}$ are alike as we have earlier discussed for the case of without AI. However, we notice the fast- declining behavior in $\mathrm{mm}$ for known and unknown $\mathrm{SD}$ as we increase the value of $\rho$. For example, when $\tau_{1}=0.3, \tau_{2}=0.29, \rho=$ 0.5 the value of $m m=12$ for $A Q L=$ 0.0025 and $L Q L=0.005$, for $\rho=0.95$, the value of $\mathrm{mm}$ is just 2. Similarly, when $A Q L=0.05, L Q L=0.10, \tau_{1}=0.5$, $\tau_{2}=0.49, \rho=0.5, m m=13$, for $\rho=0.95$ it is just 2 for known SD. For unknown SD, we observe $m m=182$ when $\rho=0.5, \tau_{1}=$ $0.5, \tau_{2}=0.49, A Q L=0.03$ and $L Q L=$ 0.055 and for $\rho=0.95$ the value of $m m=164$ keeping the rest of the values is same

Table 3. The plan parameters of suggested plan when SD is known when $\rho=0.50$

\begin{tabular}{|c|c|c|c|c|c|c|c|}
\hline & & $\tau_{1}=0.1$ & $\tau_{2}=0.09$ & $\tau_{1}=0.3$ & $\tau_{2}=0.29$ & $\tau_{1}=0.5$ & $\tau_{2}=0.49$ \\
\cline { 3 - 8 } AQL & LQL & $m m$ & $J_{a}$ & $m m$ & $J_{a}$ & $m m$ & $J_{a}$ \\
\hline 0.001 & 0.002 & 3 & 2.9576 & 14 & 2.9711 & 36 & 2.9711 \\
\hline & 0.004 & 2 & 2.7951 & 4 & 2.8219 & 9 & 2.8495 \\
\hline & 0.006 & 2 & 2.7208 & 2 & 2.7700 & 5 & 2.7659 \\
\hline & 0.008 & 2 & 2.6455 & 2 & 2.7828 & 4 & 2.7142 \\
\hline 0.0025 & 0.005 & 2 & 2.6748 & 12 & 2.6750 & 31 & 2.6766 \\
\hline & 0.010 & 2 & 2.4574 & 3 & 2.5561 & 7 & 2.5368 \\
\hline & 0.015 & 2 & 2.4523 & 2 & 2.4209 & 4 & 2.4498 \\
\hline & 0.020 & 2 & 2.6597 & 2 & 2.4803 & 3 & 2.3940 \\
\hline 0.03 & 0.06 & 2 & 1.7083 & 6 & 1.6966 & 16 & 1.6986 \\
\hline & 0.09 & 2 & 1.5161 & 3 & 1.5510 & 6 & 1.5783 \\
\hline & 0.12 & 2 & 1.5862 & 2 & 1.5357 & 4 & 1.4946 \\
\hline & 0.15 & 2 & 1.5983 & 2 & 1.3724 & 3 & 1.4069 \\
\hline 0.05 & 0.10 & 2 & 1.4836 & 5 & 1.4427 & 13 & 1.4385 \\
\hline & 0.15 & 2 & 1.3165 & 2 & 1.2817 & 5 & 1.2928 \\
\hline & 0.20 & 2 & 0.9433 & 2 & 1.1740 & 3 & 1.2038 \\
\hline & 0.25 & 2 & 1.1033 & 2 & 1.3373 & 2 & 1.1383 \\
\hline
\end{tabular}


Table 4. The plan parameters of suggested plan when SD is known when $\rho=0.950$

\begin{tabular}{|c|c|c|c|c|c|c|c|}
\hline & & $\tau_{1}=0.1$ & $\tau_{2}=0.09$ & $\tau_{1}=0.3$ & $\tau_{2}=0.29$ & $\tau_{1}=0.5$ & $\tau_{2}=0.49$ \\
\cline { 3 - 8 } AQL & LQL & $m m$ & $J_{a}$ & $m m$ & $J_{a}$ & $m m$ & $J_{a}$ \\
\hline 0.001 & 0.002 & 2 & 2.9594 & 2 & 2.9786 & 5 & 2.9686 \\
\hline & 0.004 & 2 & 2.7407 & 2 & 2.8442 & 2 & 2.8728 \\
\hline & 0.006 & 2 & 2.7358 & 2 & 2.6854 & 2 & 2.7682 \\
\hline & 0.008 & 2 & 2.7818 & 2 & 2.4912 & 2 & 2.6336 \\
\hline 0.0025 & 0.005 & 2 & 2.6596 & 2 & 2.6639 & 4 & 2.6766 \\
\hline & 0.010 & 2 & 2.4198 & 2 & 2.5658 & 2 & 2.6006 \\
\hline & 0.015 & 2 & 2.5434 & 2 & 2.2787 & 2 & 2.3840 \\
\hline & 0.020 & 2 & 2.2446 & 2 & 2.3399 & 2 & 2.5311 \\
\hline 0.01 & 0.02 & 2 & 2.1096 & 2 & 2.1740 & 3 & 2.1723 \\
\hline & 0.03 & 2 & 2.1737 & 2 & 2.1561 & 2 & 2.1143 \\
\hline & 0.04 & 2 & 2.0983 & 2 & 1.9925 & 2 & 2.0675 \\
\hline & 0.05 & 2 & 2.1783 & 2 & 2.0702 & 2 & 1.9574 \\
\hline 0.05 & 0.10 & 2 & 1.3843 & 2 & 1.4268 & 2 & 1.4590 \\
\hline & 0.15 & 2 & 1.4098 & 2 & 1.5150 & 2 & 1.1968 \\
\hline & 0.20 & 2 & 1.4551 & 2 & 1.1339 & 2 & 1.3474 \\
\hline & 0.25 & 2 & 0.7157 & 2 & 1.2959 & 2 & 1.2701 \\
\hline
\end{tabular}

Table 5. The plan parameters of suggested plan when SD is unknown and $\rho=0.50$

\begin{tabular}{|c|c|c|c|c|c|c|c|}
\hline & & $\tau_{1}=0.1$ & $\tau_{2}=0.09$ & $\tau_{1}=0.3$ & $\tau_{2}=0.29$ & $\tau_{1}=0.5$ & $\tau_{2}=0.49$ \\
\cline { 3 - 7 }$A Q L$ & $L Q L$ & $m m$ & & & & & \\
\hline 0.003 & 0.009 & 191 & 2.5365 & 194 & 2.5361 & 201 & 2.5360 \\
\hline & 0.010 & 154 & 2.5151 & 157 & 2.5151 & 163 & 2.5143 \\
\hline & 0.015 & 77 & 2.4313 & 79 & 2.4302 & 82 & 2.4313 \\
\hline & 0.020 & 51 & 2.3694 & 52 & 2.3694 & 55 & 2.3716 \\
\hline 0.015 & 0.030 & 211 & 2.0104 & 215 & 2.0100 & 227 & 2.0098 \\
\hline & 0.035 & 132 & 1.9722 & 136 & 1.9726 & 144 & 1.9721 \\
\hline & 0.040 & 93 & 1.9398 & 96 & 1.9393 & 102 & 1.9398 \\
\hline & 0.050 & 57 & 1.8849 & 58 & 1.8830 & 62 & 1.8827 \\
\hline 0.03 & 0.055 & 162 & 1.7244 & 168 & 1.7246 & 182 & 1.7240 \\
\hline & 0.057 & 142 & 1.7149 & 148 & 1.7145 & 160 & 1.7140 \\
\hline & 0.059 & 126 & 1.7058 & 131 & 1.7061 & 141 & 1.7051 \\
\hline & 0.06 & 119 & 1.7012 & 125 & 1.7019 & 133 & 1.7007 \\
\hline & 0.09 & 39 & 1.5858 & 40 & 1.5878 & 44 & 1.5850 \\
\hline 0.05 & 0.08 & 173 & 1.5123 & 182 & 1.5124 & 200 & 1.5119 \\
\hline & 0.09 & 103 & 1.4775 & 109 & 1.4773 & 120 & 1.4765 \\
\hline & 0.10 & 70 & 1.4460 & 74 & 1.4453 & 81 & 1.4453 \\
\hline & 0.11 & 51 & 1.4176 & 54 & 1.4168 & 60 & 1.4159 \\
\hline
\end{tabular}




\begin{tabular}{|l|l|l|l|l|l|l|l|}
\hline & 0.15 & 22 & 1.3189 & 23 & 1.3210 & 26 & 1.3191 \\
\hline
\end{tabular}

\section{Advantages of proposed plan}

In this section, we will examine the competence of the suggested plan with a various existing plan using with and without auxiliary information for the case of known and unknown SD. To save space, we will consider the case without AI only for $\tau_{1}=0.3, \tau_{2}=$ 0.29 for known and unknown SD. Similarly, for AI we consider the case only for $\rho=0.95, \tau_{1}=0.3, \tau_{2}=$ 0.29 and $\tau_{1}=0.5, \tau_{2}=0.49$ for known SD, and for unknown SD we use

$\rho=0.5, \tau_{1}=0.3, \tau_{2=0.29}$ and $\tau_{1}=$ $0.5, \tau_{2}=0.49$.We tabulated all the values of plan parameters in Tables 7 10. From these Tables 7-10, we observe that the proposed plan is ameliorated than the several existing plans in the form of lesser value of plan parameter for all cases using any combination of AQL and LQL. For example for the case of without AI and known $\mathrm{SD}$, using $A Q L=$
0.001, $L Q L=0.0015, \tau_{1}=0.3, \tau_{2}=$ 0.29 the value of the proposed plan is 54, for the case Khan et al. ( 2018) it was 86 , for Aslam et al. (2015b) it was 101 and for a single variable plan, it was 574. Similarly for unknown SD and without $\mathrm{AI}$, when $A Q L=$ 0.05, $L Q L=0.08, \tau_{1}=0.3, \tau_{2}=0.29$ the value of suggested plan is 185 , for the case Khan et al. (2018) the value of plan parameter was 194, for Aslam et al. (2015b) it was 198 and for a single variable plan, the value of the plan parameter do not exist. For the case of $\mathrm{AI}$ and known SD, using $A Q L=0.0025, L Q L=$ $0.0030, \tau_{1}=0.5, \tau_{2}=0.49$ and $\rho=$ 0.95 , the parametric value of the proposed plan is 61, for Aslam et al. (2018) the value was 82 and for Aslam et al. (2017) it was 240. Similarly, for the case of AI using unknown SD, when $A Q L=0.03, L Q L=0.055, \tau_{1}=$ $0.3, \tau_{2}=0.29$ and $\rho=0.5$, the parametric value of the suggested plan is 168, for Aslam et al. (2018) the value was 176 and for Aslam et al. (2017) it was 241. 
Table 6. The plan parameters of suggested plan when SD is unknown and $\rho=0.950$

\begin{tabular}{|c|c|c|c|c|c|c|c|}
\hline \multirow{2}{*}{$A Q L$} & & $\tau_{1}=0.1$ & $\tau_{2}=0.09$ & $\tau_{1}=0.3$ & $\tau_{2}=0.29$ & $\tau_{1}=0.5$ & $\tau_{2}=0.49$ \\
\cline { 3 - 7 } & $L Q L$ & $m m$ & & & & & \\
\hline 0.003 & 0.009 & 191 & 2.5360 & 192 & 2.5363 & 194 & 2.5365 \\
\hline & 0.010 & 154 & 2.5159 & 156 & 2.5150 & 158 & 2.5153 \\
\hline & 0.015 & 77 & 2.4315 & 78 & 2.4310 & 79 & 2.4305 \\
\hline & 0.020 & 51 & 2.3690 & 51 & 2.3693 & 53 & 2.3661 \\
\hline 0.015 & 0.030 & 208 & 2.0099 & 209 & 2.0097 & 211 & 2.0101 \\
\hline & 0.035 & 131 & 1.9727 & 132 & 1.9721 & 133 & 1.9729 \\
\hline & 0.040 & 93 & 1.9401 & 95 & 1.9395 & 97 & 1.9396 \\
\hline & 0.050 & 56 & 1.8835 & 57 & 1.8819 & 59 & 1.8841 \\
\hline 0.03 & 0.055 & 161 & 1.7246 & 162 & 1.7246 & 164 & 1.7248 \\
\hline & 0.057 & 142 & 1.7158 & 143 & 1.7156 & 144 & 1.7152 \\
\hline & 0.059 & 125 & 1.7059 & 126 & 1.7061 & 127 & 1.7055 \\
\hline & 0.06 & 118 & 1.7009 & 119 & 1.7007 & 120 & 1.7009 \\
\hline & 0.09 & 38 & 1.5883 & 39 & 1.5894 & 39 & 1.5882 \\
\hline & 0.08 & 173 & 1.512767 & 174 & 1.512327 & 175 & 1.5123 \\
\hline & 0.09 & 103 & 1.478186 & 103 & 1.477579 & 105 & 1.4769 \\
\hline & 0.10 & 69 & 1.445621 & 70 & 1.446434 & 71 & 1.4455 \\
\hline & 0.11 & 51 & 1.417004 & 51 & 1.41655 & 52 & 1.4171 \\
\hline & 0.15 & 21 & 1.319319 & 22 & 1.317124 & 22 & 1.3157 \\
\hline
\end{tabular}

Table 7. Comparison of plan parameter for known SD

\begin{tabular}{|c|c|c|c|c|c|}
\hline & & $\tau_{1}=0.3$ & $\tau=0.3$ & $\tau=0.3$ & $\tau=1$ \\
\hline & & $\tau_{2}=0.29$ & & & \\
\hline$p_{1}$ & $p_{2}$ & $\begin{array}{l}\text { Proposed } \\
\text { EEWMA }\end{array}$ & $\begin{array}{l}\text { Khan et al. } \\
\text { (2018) }\end{array}$ & $\begin{array}{c}\text { Aslam et } \\
\text { al. }(2015 \mathrm{~b})\end{array}$ & $\begin{array}{c}\text { Single } \\
\text { variable } \\
\text { plan }\end{array}$ \\
\hline 0.001 & 0.0015 & 54 & 86 & 101 & 574 \\
\hline 0.001 & 0.0020 & 18 & 30 & 34 & 193 \\
\hline 0.001 & 0.0025 & 10 & 17 & 19 & 108 \\
\hline 0.005 & 0.007 & 57 & 93 & 109 & 617 \\
\hline 0.005 & 0.010 & 13 & 21 & 25 & 139 \\
\hline 0.005 & 0.015 & 5 & 8 & 10 & 54 \\
\hline
\end{tabular}

Table 8. Comparison of plan parameter for unknown SD 


\begin{tabular}{|c|c|c|c|c|c|}
\hline & & $\lambda_{1}=0.3$ & $\tau=0.3$ & $\tau=0.3$ & $\tau=1$ \\
\cline { 2 - 4 } & $\lambda_{2}=0.29$ & & & \\
\hline & $p_{2}$ & $\begin{array}{c}\text { Proposed } \\
\text { EEWMA }\end{array}$ & $\begin{array}{c}\text { Khan } \text { et } \\
\text { al. }(2018)\end{array}$ & $\begin{array}{c}\text { Aslam et } \\
\text { al. }(2015 b)\end{array}$ & $\begin{array}{c}\text { Single } \\
\text { variable } \\
\text { plan }\end{array}$ \\
\hline 0.03 & 0.055 & 171 & 177 & 180 & 268 \\
\hline 0.03 & 0.058 & 141 & 146 & 148 & 222 \\
\hline 0.03 & 0.060 & 125 & 130 & 132 & 198 \\
\hline 0.05 & 0.08 & 185 & 194 & 198 & ----- \\
\hline 0.05 & 0.10 & 75 & 79 & 80 & 133 \\
\hline 0.05 & 0.015 & 23 & 25 & 25 & 44 \\
\hline
\end{tabular}

\section{Industrial application}

In this section, we will evaluate the competency of the suggested plan using industry- related data of color STN presentations. These colors are formed by amalgamating the color filters and monochrome, where every color pixel is separated by $G, B, R$ sub-pixels. This data set is used by
Aslam et al. (2012), Aslam et al. (2015b). In this study, the quality trait is the layer density of pixel. We have given that $U S L=12500 A^{0}$ and target density $12000 A^{0}$. We presume that $\alpha=0.05, \beta=0.10, A Q L=$ 0.05, $L Q L=0.11, \tau_{1}=0.3, \tau_{2}=$ 0.29 , then from Table 2 we have $L_{a}=1.4154$ and $m=55 . \quad$ The implementation of the proposed plan is as follow

Table 9. Comparison of plan parameter for known SD using AI when $\rho=0.95$

\begin{tabular}{|c|c|c|c|c|c|c|}
\hline & & \multirow[t]{2}{*}{$\tau_{1}=0.3$} & \multirow[t]{3}{*}{$\tau=0.3$} & $\tau_{1}=0.5$ & \multirow[t]{3}{*}{$\tau=0.5$} & \multirow[t]{3}{*}{$\tau=1$} \\
\hline & & & & \multirow{2}{*}{$\tau_{2}=0.49$} & & \\
\hline & & $\tau_{2}=0.29$ & & & & \\
\hline & & $\begin{array}{l}\text { Proposed } \\
\text { EEWMA }\end{array}$ & $\begin{array}{l}\text { Aslam et } \\
\text { al. (2018) }\end{array}$ & $\begin{array}{l}\text { Proposed } \\
\text { EEWMA }\end{array}$ & $\begin{array}{l}\text { Aslam et } \\
\text { al. (2018) }\end{array}$ & $\begin{array}{l}\text { Aslam et } \\
\text { al. (2017) }\end{array}$ \\
\hline$p_{1}$ & $p_{2}$ & & & & & \\
\hline \multirow[t]{3}{*}{0.0025} & 0.0030 & 23 & 43 & 61 & 82 & 240 \\
\hline & 0.0035 & 7 & 13 & 18 & 23 & 70 \\
\hline & 0.0040 & 4 & 7 & 9 & 12 & 36 \\
\hline
\end{tabular}




\begin{tabular}{|l|c|c|c|c|c|c|}
\hline 0.01 & 0.011 & 61 & 115 & 164 & 220 & ---- \\
\hline & 0.012 & 17 & 32 & 44 & 59 & 176 \\
\hline & 0.013 & 8 & 15 & 21 & 28 & 85 \\
\hline 0.05 & 0.055 & 36 & 69 & 98 & 130 & 388 \\
\hline & 0.057 & 19 & 37 & 53 & 68 & 204 \\
\hline & 0.060 & 10 & 19 & 27 & 35 & 105 \\
\hline
\end{tabular}

Table 10. Comparison of plan parameter for unknown SD using AI when $\rho=0.5$

\begin{tabular}{|c|c|c|c|c|c|c|}
\hline & & $\tau_{1}=0.3$ & $\tau=0.3$ & $\tau_{1}=0.5$ & $\tau=0.5$ & $\tau=1$ \\
\cline { 3 - 5 } & & $\tau_{2}=0.29$ & & $\tau_{2}=0.49$ & & \\
\cline { 3 - 5 }$p_{1}$ & $p_{2}$ & $\begin{array}{c}\text { Proposed } \\
\text { EEWMA }\end{array}$ & $\begin{array}{c}\text { Aslam } \text { et } \\
\text { al. }(2018)\end{array}$ & $\begin{array}{c}\text { Proposed } \\
\text { EEWMA }\end{array}$ & $\begin{array}{c}\text { Aslam } \text { et } \\
\text { al. }(2018)\end{array}$ & $\begin{array}{c}\text { Aslam } \text { et } \\
\text { al. }(2017)\end{array}$ \\
\hline 0.03 & 0.055 & 168 & 176 & 182 & 188 & 241 \\
\hline 0.03 & 0.057 & 147 & 153 & 159 & 165 & 213 \\
\hline 0.03 & 0.059 & 131 & 138 & 141 & 146 & 188 \\
\hline 0.03 & 0.060 & 123 & 128 & 134 & 138 & 179 \\
\hline 0.05 & 0.08 & 182 & 192 & 201 & 209 & ---- \\
\hline 0.05 & 0.09 & 109 & 115 & 120 & 126 & 171 \\
\hline 0.05 & 0.10 & 74 & 78 & 81 & 85 & 118 \\
\hline 0.05 & 0.11 & 54 & 57 & 59 & 62 & 87 \\
\hline
\end{tabular}

Step-1: we take a sample of size 55 from the running lot at time $i$ and compute $\overline{\bar{Z}}_{1}=11715.2$ ans $S=49.21$.

Now we suppose that $\overline{\mathrm{Z}}_{\mathrm{i}-1}=\mathrm{W}_{\mathrm{i}-1}=$ $1100, \tau_{1}=0.3, \tau_{2}=0.29$, then $\mathrm{W}_{\mathrm{i}}=11214.65$ for the current lot. Therefore, the accepting statistic $M_{i}$ is calculated as

$$
\mathrm{M}_{\mathrm{i}}=\frac{12500-11214.65}{49.21}=26.11
$$

Step-2: Accept the lot as 26.11>

\subsection{4}

\section{Concluding remarks}

We presented a memory type acceptance sampling plan using AI for known and unknown SD assuming that the main variable and AI of quality trait follow the bivariate normal distribution. We constructed the various Tables of plan parameters using different values of smoothing 
constants, different values of $\rho$ for known and unknown SD. We likened the capability of the suggested plan with numerous existing plans and exhibited that the recommended plan has a lesser value of SS for the investigation of the product. We also noticed that results were improved by using the auxiliary information. In addition, for highly correlated data, a smaller sample is needed for the inspection of the product. As the SS becomes large, the time and cost of the inspection of the commodities have increased. So it is suggested to use the proposed plan in the industry for investigating the goods to save cost and time maintaining the producer's and consumer's risk protection 


\section{Reference}

Al-Omari, A., \& Al-Hadhrami, S. (2018). Acceptance sampling plans based on truncated life tests for Extended Exponential distribution. Kuwait Journal of Science, 45(2): 3041.

Aslam, M., Azam, M., \& Jun, C.-H. (2015a). Improved acceptance

Journal of Systems Science, 46(8): 1392-1400.

Aslam, M., Azam, M., \& Jun, C.-H. (2018). Sampling plan using EWMA statistic of regression estimator. Iranian Journal of Science and Technology, Transactions A: Science, 42(1): 115-127.

Aslam, M., Huang, S.-R., Chi, H.-J., Ahmad, M., \& Rasool, M. (2011). A reliability sampling plan based on progressive interval censoring under Pareto distribution of second kind. Industrial Engineering and Management Systems, 10(2): 154-160.

Aslam, M., Satti, S. L., Moemen, M. A.-e., \& Jun, C.-H. (2017). Design of sampling plan using auxiliary information. Communications in Statistics-Theory and Methods, 46(8): 3772-3781.

Aslam, M., Wu, C.-W., Azam, M., \& Jun, C.-H. (2013). Variable sampling inspection for resubmitted lots based on process capability index $\mathrm{Cpk}$ for normally distributed items. Applied Mathematical Modelling, 37(3): 667675.
Aslam, M., Yen, C.-H., Chang, C.H., Jun, C.-H., Ahmad, M., \& Rasool, M. (2012). Two-stage variables acceptance sampling plans using process loss functions. Communications in Statistics-Theory and Methods, 41(20): 3633-3647.

Azam, M., Arif, O. H., Aslam, M., \& Ejaz, W. (2016). Repetitive acceptance sampling plan based on exponentially weighted moving average regression estimator. Journal of Computational and Theoretical Nanoscience, 13(7): 4413-4426.

Balamurali, S., \& Jun, C.-h. (2006). Repetitive group sampling procedure for variables inspection. Journal of Applied Statistics, 33(3): 327-338.

Balamurali, S., \& Jun, C.-H. (2007). Multiple dependent state sampling plans for lot acceptance based on measurement data. European Journal of Operational Research, 180(3): 1221-1230.

Cha, J. H., \& Badía, F. (2020). Variables acceptance reliability sampling plan for items subject to inverse Gaussian degradation process. Journal of Applied Statistics, 1-17.

Chakrabarty, J. B., Chowdhury, S., \& Roy, S. (2020). Optimum reliability acceptance sampling plan using TypeI generalized hybrid censoring scheme for products under warranty. International Journal of Quality \& Reliability Management. 
Collani, E. v. (1991). A note on acceptance sampling for variables. Metrika, 38(1): 19-36.

Duncan, A. (1986). Quality Control and Industrial Statistics, 5th edn (Homewood, IL, Richard D. Irwin). GALLUS, G., MANDELLI, C., MARCHI, M. \& RADAELLI, G.(1986) On surveillance methods for congenital.

Hamaker, H. C. (1979). Acceptance sampling for percent defective by variables and by attributes. Journal of Quality Technology, 11(3): 139-148.

Khan, N., Aslam, M., Ahmad, L., \& Jun, C.-H. (2019). Multiple dependent state repetitive sampling plans with or without auxiliary variable. Communications in Statistics-Simulation and Computation, 48(4): 1055-1069.

Khan, N., Aslam, M., Jun, C.-H., \& Hussain, J. (2018). Design of acceptance sampling plan using a modified EWMA statistic. Communications in Statistics-Theory and Methods, 47(12): 2881-2891.

Klufa, J. (2010). Exact calculation of the Dodge-Romig LTPD single sampling plans for inspection by variables. Statistical Papers, 51(2): 297-305.

Lieberman, G. J., \& Resnikoff, G. J. (1955). Sampling plans for inspection by variables. Journal of the American Statistical Association, 50(270): 457516.
Naveed, M., Azam, M., Khan, N., \& Aslam, M. (2018). Design of a Control Chart Using Extended EWMA Statistic. Technologies, 6(4): 108.

Owen, D. (1967). Variables sampling plans based on the normal distribution. Technometrics, 9(3): 417-423.

Roberts, S. (1959). Control chart tests based on geometric moving averages. Technometrics, 1(3): 239-250.

Seidel, W. (1997). Is sampling by variables worse than sampling by attributes? A decision theoretic analysis and a new mixed strategy for inspecting individual lots. Sankhyā: The Indian Journal of Statistics, Series $B, 96-107$.

Tripathi, H., Dey, S., \& Saha, M. (2020). Double and group acceptance sampling plan for truncated life test based on inverse log-logistic distribution. Journal of Applied Statistics, 1-16.

Wang, C.-H., \& Tsai, W. (2012). Determining the production lot size with a heuristic inspection policy for controlling the quality of input materials and products. International Journal of Systems Science, 43(11): 2030-2039.

Yemshanov, D., Haight, R. G., Liu, N., Chen, C., MacQuarrie, C. J., Ryall, K., . . . Koch, F. H. (2020). Acceptance sampling for costeffective surveillance of emerald ash borer in urban environments. Forestry: An International Journal of Forest Research, 93(2): 280-296. 
Yen, C.-H., Aslam, M., \& Jun, C.-H. (2014). A lot inspection sampling plan based on EWMA yield index. The International Journal of Advanced Manufacturing Technology, 75(5-8): 861-868.

$\begin{array}{lr}\text { Submitted: } & 01 / 06 / 2020 \\ \text { Revised: } & 15 / 10 / 2020 \\ \text { Accepted: } & 18 / 10 / 2020 \\ \text { DOI: } & 10.48129 / \text { kjs.v48i4.9860 }\end{array}$

